\title{
SEED: the six excesses (Liu Yin) evaluation and diagnosis scale
}

\author{
Pei-Jung Chiang ${ }^{1,2}$, Tsai-Chung Li ${ }^{3}$, Chih-Hung Chang ${ }^{4}$, Li-Li Chen ${ }^{5,6}$, Jun-Dai Lin ${ }^{7}$ and Yi-Chang Su ${ }^{1 *}$
}

\begin{abstract}
Background: Infections such as common colds, influenza, acute upper respiratory infections, bacterial gastroenteritis, and urinary tract infections are usually diagnosed according to patients'signs and symptoms. This study aims to develop a scale for the diagnosis of infectious diseases based on the six excesses (Liu Yin) etiological theory of Chinese medicine (CM) by the Delphi method.

Methods: A total of $200 \mathrm{CM}$-guided diagnostic items measuring signs and symptoms for infectious diseases were compiled from CM literature archives from the Han to Ming dynasties, CM textbooks in both China and Taiwan, and journal articles from the China Knowledge Resource Integrated Database. The items were based on infections and the six excesses (Liu Yin) etiological theory, i.e., Feng Xie (wind excess), Han Xie (coldness excess), Shu Xie (summer heat excess), Shi Xie (dampness excess), Zao Xie (dryness excess), and Huo Xie (fire excess). The items were further classified into the six excess syndromes and reviewed via a Delphi process to reach consensus among CM experts.
\end{abstract}

Results: In total, 178 items with a mean or median rating of 7 or above on a scale of 1-9 from a panel of 32 experts were retained. The numbers of diagnostic items in the categories of Feng (wind), Han (coldness), Shu (summer heat), Shi (dampness), Zao (dryness), and Huo (fire) syndromes were 15, 22, 25, 37, 17, and 62, respectively.

Conclusions: A CM-based six excesses (Liu Yin) evaluation and diagnosis (SEED) scale was developed for the evaluation and diagnosis of infectious diseases based only on signs and symptoms.

\section{Background}

Infections such as common colds, influenza, acute upper respiratory infections, bacterial gastroenteritis, and urinary tract infections are usually diagnosed according to patients' signs and symptoms, while the diagnosis of pandemic infections such as influenza H1N1 [1-4] and H5N1 [5, 6] must be confirmed by expensive laboratory tests [7-9] or real-time RT-PCR assays of multiple specimens [10]. Pathogen testing in the laboratory might be of low sensitivity [11], and low accuracy in some cases [1214], and above all time-consuming [15].

Chinese medicine $(\mathrm{CM})$ can detect those infectious diseases mentioned above according to the etiological theory of Liu Yin (six excesses), i.e., Feng Xie (wind excess) representing varying temperature factors, Han

\footnotetext{
*Correspondence: sychang@mail.cmu.edu.tw

${ }^{1}$ Graduate Institute of Chinese Medicine, School of Chinese Medicine,

China Medical University, Taichung, Taiwan

Full list of author information is available at the end of the article
}

Xie (coldness excess) representing falling temperature, Shu Xie (summer heat excess) representing rising temperature and humidity, Shi Xie (dampness excess) representing rising humidity, Zao Xie (dryness excess) representing falling humidity, and Huo Xie (fire excess) representing rising temperature [16, 17]. These Liu Yin (six excesses) collectively describe the circumstantial influences on $Q i$ and Xue (blood), encompassing a number of CM diagnostic criteria checked by inquiry, inspection, olfaction, audition, percussion, palpation, and pulse examination (Fig. 1), and facilitate diagnostic and therapeutic decisions [18]. However, there has been no standard diagnostic assessment or measurement scales designed for infectious diseases based on the Liu Yin (six excesses) theory [19-28].

This study aims to develop the six excesses (Liu Yin) evaluation and diagnosis (SEED) scale for infectious diseases based on the Liu Yin (six excesses) etiological 


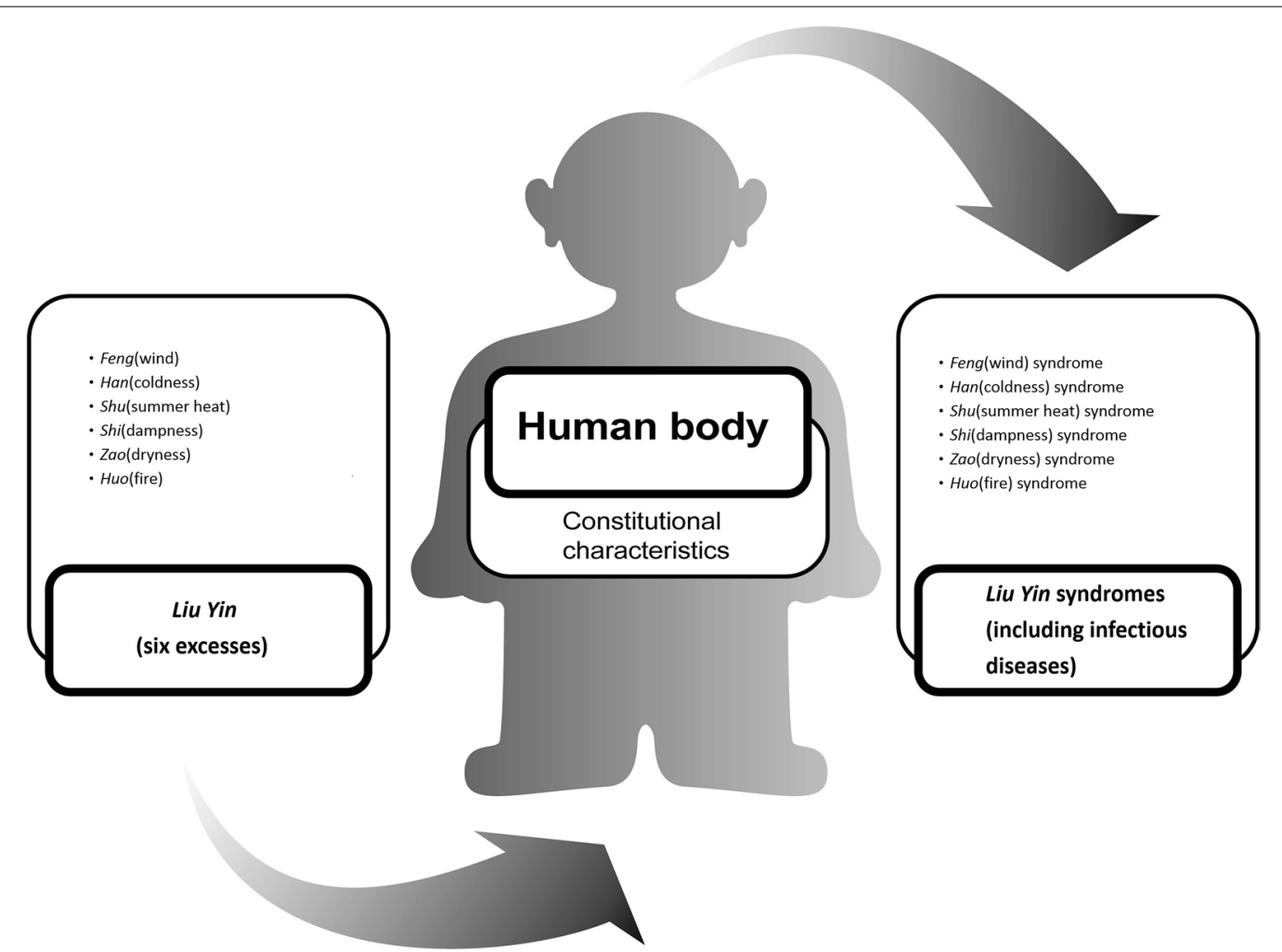

Fig. 1 Infectious diseases were classifiable in CM according to the Liu Yin (six excesses), Feng (wind), Han (coldness), Shu (summer heat), Shi (dampness), Zao (dryness), and Huo (fire), and could be classified into Feng (wind), Han (coldness), Shu (summer heat), Shi (dampness), Zao (dryness), and Huo (fire) syndromes

theory in CM by a Delphi process among experts. The Delphi method aims to build consensus and generate ideas in research fields [29-41], and is useful for the establishment of diagnostic criteria in clinical medicine. It is a structured communication process to establish the definition of the syndromes, the diagnostic criteria or the staging of the diseases, and the suggested treatments in the medical guidelines [42-48].

\section{Methods}

The Delphi method [49] was used to achieve a group panel consensus on the diagnostic items for the Liu Yin (six excesses) syndromes among a panel of experts between 2007 and 2008. A nationally representative panel of Chinese medical experts were invited; only CM experts with good knowledge about $\mathrm{CM}$ and modern research methods with master or doctoral degrees, and more than 7 years of practicing experience were invited to join the Delphi panel. Based on this consensus, we carried out further statistical analyses for infectious diseases.

An interdisciplinary advisory board was formed by seven members, including five CM experts, one measurement methodologist, and one statistician. The advisory board selected the participants of the Delphi panel.
Representatives from various education backgrounds, medical disciplines, geographical distributions, and clinical experience were considered for the panel. Finally, a total of $32 \mathrm{CM}$ experts meeting these criteria were invited, and all agreed to participate (Table 1). Of the 32 participants, 20 were from CM departments in medical centers, 6 were from district or regional teaching hospitals, and 6 were from private practices; 7 practiced in Northern region, 11 in central region, 6 in Southern region and 8 in other region. Twenty-one panelists held master degrees, 11 had doctoral degrees in medical sciences, and 26 panelists were teachers in academic institutions. The age (mean \pm SD) of the panelists was $43 \pm 7.0$ years with a median of 42 years. The year of practicing experience (mean \pm SD) was $11.3 \pm 3.7$ years with a median of 10 years.

The Delphi process was iterative. We began with a systematic review of traditional CM literature including the Medicine Encyclopedia collected by Kentang Wang (AC1552-1639), 122 published modern textbooks, and 7364 journal articles from the China Knowledge Resource Integrated Database. The search keywords included: Feng (wind), Han (coldness), Shu (summer heat), Shi (dampness), Zao (dryness), Huo (fire), Wai-gan (external contraction), Liu Yin (six excesses), and Yin (excess), and 
Table 1 Basic characteristics of the $32 \mathrm{CM}$ experts

\begin{tabular}{ll}
\hline Age, mean (SD), years & \\
Average & $43(\mathrm{SD}=7.0)$ \\
Median & 42 \\
Spectrum of practice, mean (SD), years & \\
Average & $11.3(\mathrm{SD}=3.7)$ \\
Median & 10 \\
Educational background, $\mathrm{n}(\%)$ & \\
Master degree & $21(65.6 \%)$ \\
Doctorate degree & $11(34.4 \%)$ \\
Geographic distribution, $\mathrm{n}(\%)$ & \\
Northern & $7(21.9 \%)$ \\
Central & $11(34.4 \%)$ \\
Southern & $6(18.8 \%)$ \\
Other & $8(25.0 \%)$ \\
Practice institution, $\mathrm{n}(\%)$ & \\
Medical centers & $20(62.5 \%)$ \\
District teaching hospitals & $5(15.6 \%)$ \\
Regional teaching hospitals & $1(3.1 \%)$ \\
Private practices & $6(18.8 \%)$ \\
Teaching in an academic institution, $\mathrm{n}(\%)$ & \\
Yes & $26(81.3 \%)$ \\
No & $6(18.8 \%)$ \\
\hline & \\
\hline &
\end{tabular}

we identified and compiled a pool of 200 diagnostic items for the Liu Yin (six excesses) syndromes. The CM experts categorized each of these 200 items into one of the Liu Yin (six excesses) syndromes, and the results were reviewed and face-validated by the same experts. The numbers of items for each Yin (excess) syndrome were: Feng (wind), 23; Han (coldness), 32; Shu (summer heat), 25; Shi (dampness), 40; Zao (dryness), 18; and Huo (fire), 62. We had mailed the items to the panelists and invited them to add any items; the numbers remained the same.

The classified items were then incorporated into the processes of the Delphi method and circulated via mail to the panelists for their ratings with follow-up phone calls within 2 weeks (Fig. 2). In stage 1, the panelists were asked to rate the appropriateness of each of the classified signs and symptoms on a scale of 1 (highly inappropriate) to 9 (highly appropriate). They were also instructed to provide reasons for the items they rated as "inappropriate". In stage 2 , the results for the mean rating and standard deviation of the individual items were mailed to the panelists, and they were asked again to provide and return their ratings after reviewing the results. The two-stage Delphi method are enough to saturate consensus [49-57], which cannot be maximized by increasing the number of round [58-64]. The progress was assessed by any reduction in

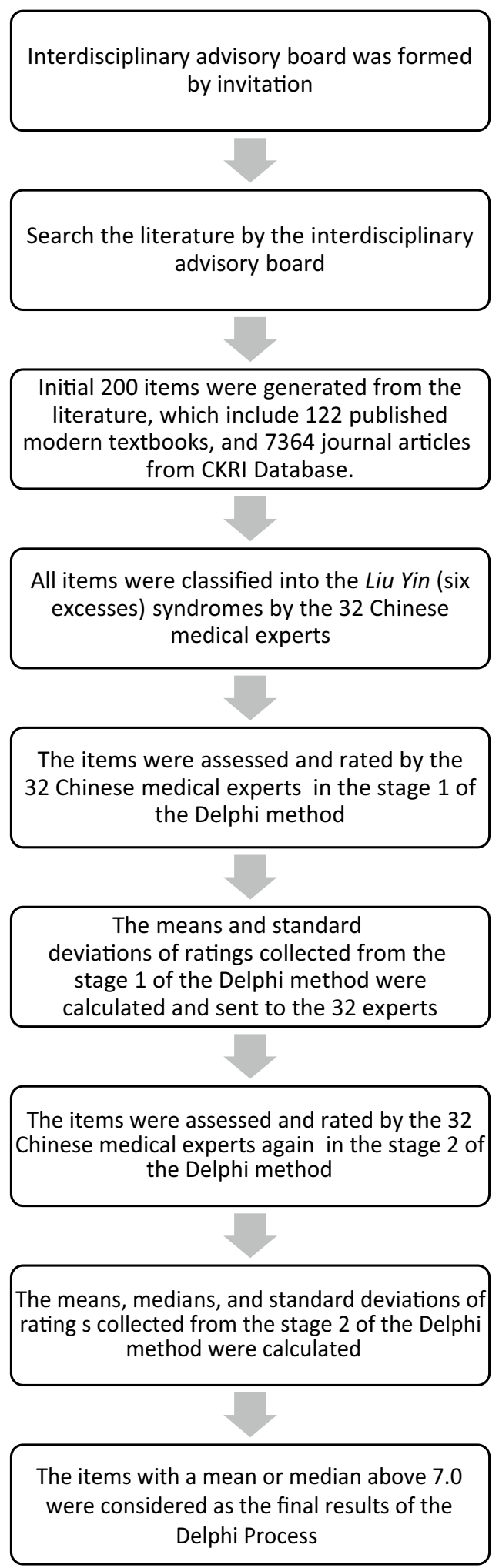

Fig. 2 Flow chart of the Delphi process 
the variability of judgments among the panelists. The level of consensus was quantified by the standard deviation of their ratings. After the two-stage Delphi method, the expert-rated diagnostic items with an average or median rating of 7 or above were considered to have face-validity and integrated into the standardized assessment. The cut point of 7.0 had been chosen because it highly represents panelists' agreement as well as the estimated time required to complete the selected items by $\mathrm{CM}$ doctors is expected to be no more than $30 \mathrm{~min}$.

\section{Results}

The mean, median and standard deviation of the ratings for each item from the first and second stages were calculated (Table 2). After the two-stage Delphi method, 15, 22, $25,37,17$, and 62 diagnostic items with a rating of 7.0 or higher were retained in Feng (wind), Han (coldness), Shu (summer heat), Shi (dampness), Zao (dryness), and Huo (fire) syndromes, respectively. The standard deviations of all 178 retained items decreased from the first stage to the second stage because of the achieved agreement, with the following exceptions: "surging but weak pulse with dipped finger tip" (both 1.02) and "unclear head and eyesight" (1.01-1.06) in Shu (summer heat) syndrome; "edematous swelling in face and limbs" (both 1.10) and "unclear head and eyesight" (both 0.67) in Shi (dampness) syndrome; and "dry and yellow fur" (1.01-1.06) and "dry, yellow and white fur" (0.61-0.63) in Huo (fire) syndrome. However, the upward changes were relatively small.

The total number of retained items in each of the Liu Yin (six excesses) syndromes varied from 15 items in Feng (wind) syndrome to 62 items in Huo (fire) syndrome. All syndromes comprised two kinds of diagnostic items: subjective discomfort factors and objective examinations by the patients themselves or by clinicians. The diagnostic items for subjective discomfort included the following: items regarding sleeping, appetite, eyesight, and hearing; items regarding behavioral adjustments such as aversion, preference, anguish, and agitation; items regarding sensations of coldness, hotness, dryness, and bitterness; and items regarding feelings of pain, itch, ache, congestion, thirst, viscousness, oppression, fullness, stuffiness, heaviness, and tiredness. The diagnostic items for objective examinations included the following: general symptoms regarding complexion, eyes, lips, tongue, skin, snivel, stool, urine, phlegm, and awareness; physical responses such as breathlessness, coughing, shivering, sweating, sneezing, and vomiting; and appearance changes such as hemiplegia, deviated eyes and mouth, opisthotonos, trismus, and edema. In addition to the two kinds of diagnostic items, $4,2,3,4$, and 5 items of pulse examination were retained in Feng (wind), Han (coldness), Shu (summer heat), Shi (dampness), and Huo (fire) syndromes, respectively.
Some items were retained in more than one syndrome because of their overlapping contributions, as follows: "headache", "generalized pain", "clear snivel", and "headache and painful stiffness in nape" in Feng (wind) and Han (coldness) syndromes; "floating and rapid pulse" in Feng (wind) and Huo (fire) syndromes; "aches and pains in joints" and "a lot of clear and white phlegm" in Han (coldness) and Shi (dampness) syndromes; "fatigue", "heavy or tired limbs", and "unclear head and eyesight" in Shu (summer heat) and Shi (dampness) syndromes; "thirsty", "desire to drink", and "dry tongue with little saliva" in Shu (summer heat), Zao (dryness), and Huo (fire) syndromes; "dry nasal cavity", "dry lips", "dry throat", "hard bound or dry stool", and "dry, yellow and white fur" in Zao (dryness) and Hио (fire) syndromes; and "aversion to heat", "fever", "high fever", "profuse sweating", "red lips", "preference for cold drinks", "agitation", "shortness of urine", "reddish yellow urine", "red tongue", "surging pulse", and "rapid pulse" in Shu (summer heat) and Huo (fire) syndromes. Including these overlapping items, the SEED scale for infectious diseases, which captured the Liu Yin (six excesses) syndromes, was created and formatted as follows: Feng (wind), 15 items; Han (coldness), 22 items; Shu (summer heat), 25 items; Shi (dampness), 37 items; Zao (dryness), 17 items; and Нио (fire), 62 items. Following the integration of common items, a total of 102 diagnostic criteria conformed to the Liu Yin (six excesses) and covered the manifestations of the Liu Yin (six excesses) syndromes.

\section{Discussion}

This study codified the Liu Yin (six excesses) syndromes by the Delphi method with a panel of $32 \mathrm{CM}$ experts. The 178 diagnostic items derived from the two-stage Delphi method combined information gleaned from CM classic textbooks, journal articles, and opinions of CM experts. All six syndromes encompassed diagnostic criteria regarding subjective discomfort and objective examinations, both were deemed essential for diagnostic judgment. Subjective discomfort was important for medical care considerations, while objective examinations were crucial for disease progress evaluations.

Common diagnostic items were present in different syndromes as a result of the same body responses to different Yin (excess). For example, "generalized pain" in Feng (wind) and Shi (dampness) syndromes results from obstructed circulation of $Q i$ and Xue (blood) [65]. Common items combined with different signs or symptoms imply different body responses [66, 67]. "Fatigue" combined with "thirsty" was attributed to Shu Xie (summer heat excess), while "fatigue" combined with "not thirsty" was attributed to Shi Xie (dampness excess).

Five syndromes, except for Feng (wind) syndrome, comprised diagnostic items of tongue examinations for 
Table 2 Results of the two-stage Delphi method

\begin{tabular}{|c|c|c|c|c|c|c|}
\hline \multirow[t]{2}{*}{ Syndrome } & \multirow[t]{2}{*}{ No. } & \multirow[t]{2}{*}{ Item } & \multicolumn{2}{|l|}{ Stage 1} & \multicolumn{2}{|l|}{ Stage 2} \\
\hline & & & Mean (SD) & Median & Mean (SD) & Median \\
\hline \multirow[t]{23}{*}{ Feng (wind) syndrome } & 1 & Aversion to wind ${ }^{\mathrm{a}}$ & $8.03(1.06)$ & 8 & $8.28(0.96)$ & 8 \\
\hline & 2 & Floating pulse $\mathrm{a}^{\mathrm{a}}$ & $7.63(1.41)$ & 7 & $7.78(0.87)$ & 7 \\
\hline & 3 & Itchy throat ${ }^{\mathrm{a}}$ & $7.25(1.16)$ & 7 & $7.41(0.80)$ & 7 \\
\hline & 4 & Headache $e^{\mathrm{a}}$ & $7.09(1.30)$ & 6 & $7.16(0.51)$ & 6.5 \\
\hline & 5 & Headache and painful stiffness in nape & $7.06(1.34)$ & 7 & $7.06(0.80)$ & 7 \\
\hline & 6 & Floating and relaxed pulse $\mathrm{e}^{\mathrm{a}}$ & $7.00(1.42)$ & 6 & $7.28(0.82)$ & 7 \\
\hline & 7 & Clear snivel $^{a}$ & $6.97(1.23)$ & 7 & $7.09(0.69)$ & 7 \\
\hline & 8 & Sneezing ${ }^{a}$ & $6.94(1.34)$ & 5 & $7.13(0.79)$ & 5 \\
\hline & 9 & Pain at unfixed location & $6.88(2.03)$ & 6 & $6.91(1.09)$ & 6 \\
\hline & 10 & Painful stiffness in nape and back & $6.84(1.22)$ & 6 & $6.84(0.95)$ & 5 \\
\hline & 11 & Floating and rapid pulse $\mathrm{e}^{\mathrm{a}}$ & $6.81(1.42)$ & 7 & $7.19(0.82)$ & 7 \\
\hline & 12 & Generalized pain ${ }^{\mathrm{a}}$ & $6.75(1.59)$ & 7 & $7.06(0.67)$ & 7 \\
\hline & 13 & Nasal congestion ${ }^{\mathrm{a}}$ & $6.69(1.31)$ & 7 & $7.13(0.75)$ & 7 \\
\hline & 14 & Cough, productive ${ }^{a}$ & $6.59(1.46)$ & 7 & $7.06(0.67)$ & 7 \\
\hline & 15 & Floating and weak pulse $\mathrm{a}^{\mathrm{a}}$ & $6.53(1.65)$ & 7 & $6.59(1.07)$ & 7 \\
\hline & 16 & Aches and pains in joints & $6.44(1.39)$ & 5.5 & $6.69(0.59)$ & 5 \\
\hline & 17 & Generalized itching & $6.44(1.68)$ & 5.5 & $6.44(1.37)$ & 5 \\
\hline & 18 & Thin white fur & $6.38(1.79)$ & 6 & $6.50(1.05)$ & 6 \\
\hline & 19 & Pale red tongue & $6.28(1.90)$ & 6 & $6.41(0.98)$ & 6 \\
\hline & 20 & Deviated eye and mouth & $6.16(1.92)$ & 8 & $5.84(1.44)$ & 8 \\
\hline & 21 & Opisthotonos $^{\mathrm{a}}$ & $5.63(2.23)$ & 7 & $5.09(1.40)$ & 7 \\
\hline & 22 & Trismus (lockjaw) ${ }^{a}$ & $5.53(2.17)$ & 7 & $5.06(1.37)$ & 7 \\
\hline & 23 & Hemiplegia & $5.22(2.35)$ & 6 & $5.13(1.43)$ & 6 \\
\hline \multirow[t]{25}{*}{ Han (coldness) syndrome } & 1 & Aversion to cold ${ }^{\mathrm{a}}$ & $8.22(0.87)$ & 8.5 & $8.41(0.61)$ & 8 \\
\hline & 2 & Floating and tight pulse $\mathrm{a}^{\mathrm{a}}$ & $7.66(1.10)$ & 7 & $7.78(0.79)$ & 7 \\
\hline & 3 & Preference for hot drinks ${ }^{a}$ & $7.53(1.37)$ & 7 & $7.53(0.67)$ & 7 \\
\hline & 4 & Clear phlegm ${ }^{a}$ & $7.53(0.98)$ & 7 & $7.47(0.57)$ & 7 \\
\hline & 5 & Clear snivel $^{\mathrm{a}}$ & $7.41(0.95)$ & 6 & $7.50(0.67)$ & 7 \\
\hline & 6 & A lot of clear and white phlegm ${ }^{a}$ & $7.38(1.07)$ & 7 & $7.34(0.60)$ & 7 \\
\hline & 7 & Generalized pain ${ }^{\mathrm{a}}$ & $7.13(1.34)$ & 7 & $7.41(0.67)$ & 7 \\
\hline & 8 & Headache and painful stiffness in nape ${ }^{a}$ & $7.13(1.18)$ & 7 & $7.28(0.58)$ & 7 \\
\hline & 9 & Shivering $^{\mathrm{a}}$ & $7.06(1.37)$ & 7 & $7.22(0.91)$ & 7 \\
\hline & 10 & Ache in limbs ${ }^{a}$ & $7.00(1.39)$ & 7 & $7.28(0.73)$ & 7 \\
\hline & 11 & Cold body and limbs ${ }^{a}$ & $7.00(1.87)$ & 7 & $7.28(0.96)$ & 7 \\
\hline & 12 & Tight pulse ${ }^{a}$ & $6.94(1.39)$ & 6 & $7.13(1.16)$ & 6 \\
\hline & 13 & Aches and pains in joints ${ }^{\mathrm{a}}$ & $6.88(1.50)$ & 6 & $7.28(0.58)$ & 6 \\
\hline & 14 & White and moist fur & $6.88(1.50)$ & 6 & $6.81(1.00)$ & 6 \\
\hline & 15 & Not thirsty ${ }^{a}$ & $6.84(1.32)$ & 6 & $7.03(0.93)$ & 6 \\
\hline & 16 & White, moist and thin fur ${ }^{\mathrm{a}}$ & $6.84(1.51)$ & 7 & $6.94(0.95)$ & 7 \\
\hline & 17 & No desire to drink & $6.84(1.27)$ & 6 & $6.81(0.90)$ & 6 \\
\hline & 18 & Clear urine $^{a}$ & $6.81(1.64)$ & 7 & $6.78(0.75)$ & 7 \\
\hline & 19 & Painful stiffness in nape and back ${ }^{a}$ & $6.78(1.72)$ & 7 & $6.91(0.95)$ & 7 \\
\hline & 20 & White and thin fur & $6.78(1.72)$ & 6 & $6.84(0.95)$ & 6 \\
\hline & 21 & Absence of sweating ${ }^{\mathrm{a}}$ & $6.69(1.64)$ & 7 & $7.13(1.01)$ & 7 \\
\hline & 22 & Cold feeling on nape and back & $6.69(1.45)$ & 7 & $6.94(0.76)$ & 6 \\
\hline & 23 & Headache $^{a}$ & $6.63(1.36)$ & 7 & $7.06(0.95)$ & 8 \\
\hline & 24 & Cough, productive & $6.53(1.24)$ & 7 & $6.75(0.72)$ & 6 \\
\hline & 25 & White fur & $6.53(1.78)$ & 7 & $6.59(1.01)$ & 6 \\
\hline
\end{tabular}


Table 2 continued

\begin{tabular}{|c|c|c|c|c|c|c|}
\hline \multirow[t]{2}{*}{ Syndrome } & \multirow[t]{2}{*}{ No. } & \multirow[t]{2}{*}{ Item } & \multicolumn{2}{|l|}{ Stage 1} & \multicolumn{2}{|l|}{ Stage 2} \\
\hline & & & Mean (SD) & Median & Mean (SD) & Median \\
\hline & 26 & Sloppy stool & $6.50(1.32)$ & 7 & $6.66(1.00)$ & 6 \\
\hline & 27 & Reversal cold of extremities & $6.50(1.80)$ & 7 & $6.59(1.24)$ & 6 \\
\hline & 28 & White and slippery fur ${ }^{a}$ & $6.44(1.63)$ & 7 & $6.50(1.02)$ & 7 \\
\hline & 29 & Nasal congestion & $6.38(1.36)$ & 7 & $6.81(0.63)$ & 6 \\
\hline & 30 & Sneezing & $6.38(1.34)$ & 6 & $6.81(0.64)$ & 6 \\
\hline & 31 & Lumbar pain or ache $e^{a}$ & $6.13(1.54)$ & 8 & $6.53(0.67)$ & 8 \\
\hline & 32 & Breathlessness ${ }^{\mathrm{a}}$ & $5.88(1.52)$ & 7 & $6.22(1.10)$ & 7 \\
\hline \multirow[t]{25}{*}{ Shu (summer heat) syndrome } & 1 & Thirsty ${ }^{a}$ & $7.97(1.00)$ & 8 & $7.88(0.71)$ & 8 \\
\hline & 2 & Fever $^{a}$ & $7.91(1.09)$ & 8 & $8.00(0.80)$ & 8 \\
\hline & 3 & High fever ${ }^{a}$ & $7.84(1.05)$ & 8 & $7.88(0.66)$ & 8 \\
\hline & 4 & Profuse sweating ${ }^{a}$ & $7.81(1.57)$ & 8 & $7.53(0.92)$ & 7 \\
\hline & 5 & Surging pulse $\mathrm{e}^{\mathrm{a}}$ & $7.66(0.94)$ & 8 & $7.66(0.79)$ & 7 \\
\hline & 6 & Fatigue $e^{\mathrm{a}}$ & $7.59(1.21)$ & 7 & $7.72(0.73)$ & 7 \\
\hline & 7 & Aversion to heat ${ }^{\mathrm{a}}$ & $7.56(1.41)$ & 7 & $7.72(0.73)$ & 7 \\
\hline & 8 & Reddish yellow urine ${ }^{a}$ & $7.56(0.88)$ & 7 & $7.47(0.67)$ & 7 \\
\hline & 9 & Red tongue $^{a}$ & $7.53(0.92)$ & 6 & $7.63(0.66)$ & 6 \\
\hline & 10 & Surging but weak pulse with dipped finger tip ${ }^{a}$ & $7.53(1.02)$ & 6 & $7.28(1.02)$ & 6 \\
\hline & 11 & Desire to drink ${ }^{\mathrm{a}}$ & $7.47(1.41)$ & 7 & $7.53(0.67)$ & 7 \\
\hline & 12 & Unclear head and eyesight $^{a}$ & $7.44(1.01)$ & 7 & $7.03(1.06)$ & 7 \\
\hline & 13 & Rapid pulse ${ }^{a}$ & $7.34(1.29)$ & 8 & $7.28(0.68)$ & 8 \\
\hline & 14 & Heavy or tired limbs ${ }^{\mathrm{a}}$ & $7.31(1.38)$ & 8 & $7.25(0.84)$ & 7 \\
\hline & 15 & Reddened complexion ${ }^{a}$ & $7.28(0.85)$ & 7 & $7.22(0.61)$ & 7 \\
\hline & 16 & Preference for cold drinks ${ }^{a}$ & $7.28(1.51)$ & 7 & $7.13(0.87)$ & 7 \\
\hline & 17 & Shortness of urine $e^{a}$ & $7.22(1.04)$ & 6 & $7.06(0.95)$ & 6 \\
\hline & 18 & Red lips ${ }^{a}$ & $7.19(0.74)$ & 7 & $7.19(0.64)$ & 6 \\
\hline & 19 & Agitation $^{a}$ & $7.13(1.04)$ & 7 & $7.00(0.76)$ & 7 \\
\hline & 20 & Lethargy $^{a}$ & $7.03(1.26)$ & 7 & $6.78(0.87)$ & 7 \\
\hline & 21 & Dry tongue with little saliva ${ }^{a}$ & $7.00(0.95)$ & 8 & $7.09(0.93)$ & 7 \\
\hline & 22 & Anorexia $^{a}$ & $6.81(1.15)$ & 8 & $6.81(0.90)$ & 8 \\
\hline & 23 & Dizziness $^{\mathrm{a}}$ & $6.50(1.22)$ & 8 & $6.47(1.08)$ & 8 \\
\hline & 24 & Stomach reflux ${ }^{a}$ & $6.38(1.07)$ & 7 & $6.34(0.90)$ & 7 \\
\hline & 25 & Headache $e^{a}$ & $6.19(1.51)$ & 7 & $6.38(1.10)$ & 7 \\
\hline \multirow[t]{16}{*}{ Shi (dampness) syndrome } & 1 & Heavy or tired limbs ${ }^{a}$ & $8.19(0.86)$ & 7 & $8.09(0.73)$ & 7 \\
\hline & 2 & Heavy-headedness ${ }^{\mathrm{a}}$ & $8.09(0.86)$ & 8 & $8.06(0.76)$ & 8 \\
\hline & 3 & Fatigue $^{a}$ & $7.81(0.90)$ & 7 & $7.81(0.74)$ & 7 \\
\hline & 4 & White and slimy fur ${ }^{\mathrm{a}}$ & $7.75(1.05)$ & 7 & $7.72(0.68)$ & 7 \\
\hline & 5 & Unclear head and eyesight ${ }^{\mathrm{a}}$ & $7.50(0.67)$ & 8 & $7.50(0.67)$ & 8 \\
\hline & 6 & Viscous feeling in oral cavity ${ }^{a}$ & $7.50(0.95)$ & 7 & $7.47(0.62)$ & 7 \\
\hline & 7 & Thick and slimy fur ${ }^{\mathrm{a}}$ & $7.47(1.16)$ & 7 & $7.63(0.79)$ & 7 \\
\hline & 8 & Soggy pulse $\mathrm{e}^{\mathrm{a}}$ & $7.41(1.34)$ & 8 & $7.47(1.02)$ & 8 \\
\hline & 9 & Edematous swelling in face and limbs ${ }^{a}$ & $7.41(1.10)$ & 7 & $7.13(1.10)$ & 7 \\
\hline & 10 & Edema, generalized & $7.28(1.53)$ & 6 & $6.94(0.95)$ & 6 \\
\hline & 11 & Rash & $7.25(1.30)$ & 6 & $6.97(1.09)$ & 6 \\
\hline & 12 & Sloppy stool ${ }^{\mathrm{a}}$ & $7.22(1.21)$ & 7 & $7.38(0.61)$ & 7 \\
\hline & 13 & Slippery pulse ${ }^{a}$ & $7.19(1.06)$ & 7 & $7.22(0.79)$ & 7 \\
\hline & 14 & Soggy and relaxed pulse $\mathrm{e}^{\mathrm{a}}$ & $7.19(1.35)$ & 7 & $7.19(0.90)$ & 7 \\
\hline & 15 & White and slippery fur ${ }^{\mathrm{a}}$ & $7.19(1.40)$ & 6 & $7.19(0.74)$ & 6.5 \\
\hline & 16 & No desire to drink $k^{a}$ & $7.19(1.23)$ & 6 & $7.00(0.88)$ & 7 \\
\hline
\end{tabular}


Table 2 continued

\begin{tabular}{|c|c|c|c|c|c|c|}
\hline \multirow[t]{2}{*}{ Syndrome } & \multirow[t]{2}{*}{ No. } & \multirow[t]{2}{*}{ Item } & \multicolumn{2}{|l|}{ Stage 1} & \multicolumn{2}{|l|}{ Stage 2} \\
\hline & & & Mean (SD) & Median & Mean (SD) & Median \\
\hline & 17 & Yellow and slimy fur ${ }^{\mathrm{a}}$ & $7.19(1.18)$ & 7 & $6.97(1.03)$ & 7 \\
\hline & 18 & Slippery fur ${ }^{\mathrm{a}}$ & $7.13(1.43)$ & 7 & $7.16(0.72)$ & 7 \\
\hline & 19 & Soft stool ${ }^{a}$ & $6.97(1.36)$ & 6 & $7.22(0.61)$ & 7 \\
\hline & 20 & Anorexia $^{a}$ & $6.97(1.40)$ & 6 & $7.22(0.71)$ & 6 \\
\hline & 21 & Dizziness $^{\mathrm{a}}$ & $6.94(1.46)$ & 7 & $7.13(0.83)$ & 7 \\
\hline & 22 & Lethargy $^{\text {a }}$ & $6.94(1.34)$ & 7 & $7.09(0.89)$ & 7 \\
\hline & 23 & White and moist fur ${ }^{\mathrm{a}}$ & $6.94(1.32)$ & 7 & $6.97(0.78)$ & 7 \\
\hline & 24 & Stool with discharge $\mathrm{e}^{\mathrm{a}}$ & $6.94(1.32)$ & 7 & $6.94(0.72)$ & 7 \\
\hline & 25 & Oppression in chest $\mathrm{t}^{\mathrm{a}}$ & $6.91(1.42)$ & 7 & $7.13(0.83)$ & 7 \\
\hline & 26 & White and moist fur ${ }^{\mathrm{a}}$ & $6.91(1.53)$ & 7 & $7.13(0.75)$ & 7 \\
\hline & 27 & Not thirsty ${ }^{\mathrm{a}}$ & $6.91(1.17)$ & 7 & $6.97(0.78)$ & 7 \\
\hline & 28 & Soggy and rapid pulse & $6.91(1.23)$ & 7 & $6.97(0.90)$ & 7 \\
\hline & 29 & Dyspepsia & $6.91(1.42)$ & 7 & $6.81(1.00)$ & 6 \\
\hline & 30 & Jaundice ${ }^{a}$ & $6.91(1.47)$ & 7 & $6.78(1.16)$ & 7 \\
\hline & 31 & Mild fever ${ }^{a}$ & $6.88(1.31)$ & 7 & $7.09(0.59)$ & 7 \\
\hline & 32 & Stomach reflux $x^{a}$ & $6.78(1.24)$ & 8 & $6.84(0.81)$ & 8 \\
\hline & 33 & Gastric stuffiness $^{a}$ & $6.75(1.46)$ & 7 & $7.06(0.76)$ & 7 \\
\hline & 34 & Vaginal discharge $\mathrm{e}^{\mathrm{a}}$ & $6.75(1.46)$ & 8 & $7.06(0.76)$ & 8 \\
\hline & 35 & A lot of clear and white phlegm ${ }^{a}$ & $6.75(1.34)$ & 7 & $7.00(0.51)$ & 7 \\
\hline & 36 & Aches and pains in joints ${ }^{\mathrm{a}}$ & $6.72(1.35)$ & 7 & $7.03(0.47)$ & 7 \\
\hline & 37 & White fur ${ }^{\mathrm{a}}$ & $6.66(1.49)$ & 7 & $6.91(0.78)$ & 7 \\
\hline & 38 & Vomiting $^{\mathrm{a}}$ & $6.59(1.24)$ & 7 & $6.56(0.98)$ & 7 \\
\hline & 39 & Ache in limbs ${ }^{a}$ & $6.50(1.32)$ & 7 & $6.72(0.58)$ & 7 \\
\hline & 40 & Generalized pain $^{\mathrm{a}}$ & $6.44(1.48)$ & 6 & $6.75(0.50)$ & 7 \\
\hline \multirow[t]{18}{*}{ Zao (dryness) syndrome } & 1 & Dry throat ${ }^{\mathrm{a}}$ & $8.16(0.72)$ & 8 & $8.17(0.66)$ & 8 \\
\hline & 2 & Dry nasal cavity ${ }^{\mathrm{a}}$ & $8.06(0.84)$ & 7 & $7.90(0.49)$ & 6 \\
\hline & 3 & Dry lips ${ }^{a}$ & $8.06(0.88)$ & 8 & $7.79(0.49)$ & 8 \\
\hline & 4 & Dry skin ${ }^{\mathrm{a}}$ & $7.84(0.92)$ & 8 & $7.83(0.54)$ & 8 \\
\hline & 5 & Dry tongue with little fluida & $7.75(1.05)$ & 8 & $7.66(0.55)$ & 8 \\
\hline & 6 & Cough, nonproductive ${ }^{\mathrm{a}}$ & $756(1.08)$ & 6 & $7.62(0.62)$ & 6 \\
\hline & 7 & Thirsty ${ }^{\mathrm{a}}$ & $7.38(1.43)$ & 8 & $7.24(0.83)$ & 7.5 \\
\hline & 8 & Hard bound or dry stool ${ }^{\mathrm{a}}$ & $7.31(1.28)$ & 7 & $7.14(0.49)$ & 7 \\
\hline & 9 & Sticky phlegm a & $7.31(1.03)$ & 7 & $7.14(0.52)$ & 7 \\
\hline & 10 & Dry, thin and white fur ${ }^{\mathrm{a}}$ & $7.25(1.34)$ & 7 & $7.17(0.80)$ & 7 \\
\hline & 11 & Dry, yellow and white fur ${ }^{\mathrm{a}}$ & $7.25(1.16)$ & 8 & $7.00(0.89)$ & 8 \\
\hline & 12 & Dry and yellow fur ${ }^{\mathrm{a}}$ & $7.06(1.29)$ & 7 & $6.79(0.73)$ & 7 \\
\hline & 13 & Desire to drink ${ }^{\mathrm{a}}$ & $7.00(1.37)$ & 7 & $7.10(0.86)$ & 7 \\
\hline & 14 & Red tongue & $6.97(1.58)$ & 6 & $6.90(0.62)$ & 6 \\
\hline & 15 & Hoarseness $^{\mathrm{a}}$ & $6.88(1.21)$ & 7 & $7.00(0.53)$ & 7 \\
\hline & 16 & Pruritus $^{\mathrm{a}}$ & $6.81(1.38)$ & 7 & $7.10(0.41)$ & 7 \\
\hline & 17 & Dyschezia $^{a}$ & $6.50(1.34)$ & 7 & $6.59(0.95)$ & 7 \\
\hline & 18 & Cough, productive & $6.28(1.17)$ & 7 & $6.59(0.82)$ & 7 \\
\hline \multirow[t]{6}{*}{ Huo (fire) syndrome } & 1 & High fever ${ }^{\mathrm{a}}$ & $7.97(0.82)$ & 8 & $8.00(0.57)$ & 8 \\
\hline & 2 & Fever $^{\mathrm{a}}$ & $7.94(1.08)$ & 8 & $7.97(0.54)$ & 8 \\
\hline & 3 & Rapid pulse & $7.94(1.19)$ & 8 & $7.84(0.68)$ & 8 \\
\hline & 4 & Heat intolerance $\mathrm{a}^{\mathrm{a}}$ & $7.91(0.86)$ & 7 & $7.94(0.72)$ & 7 \\
\hline & 5 & Thirsty $^{\mathrm{a}}$ & $7.91(1.06)$ & 7 & $7.78(0.61)$ & 7 \\
\hline & 6 & Red tongue $^{\mathrm{a}}$ & $7.88(1.01)$ & 7 & $7.75(0.67)$ & 7 \\
\hline
\end{tabular}


Table 2 continued

\begin{tabular}{|c|c|c|c|c|c|c|}
\hline \multirow[t]{2}{*}{ Syndrome } & \multirow[t]{2}{*}{ No. } & \multirow[t]{2}{*}{ Item } & \multicolumn{2}{|l|}{ Stage 1} & \multicolumn{2}{|l|}{ Stage 2} \\
\hline & & & Mean (SD) & Median & Mean (SD) & Median \\
\hline & 7 & Preference for cold drinks ${ }^{a}$ & $7.69(1.00)$ & 6 & $7.63(0.66)$ & 6 \\
\hline & 8 & Surging pulse & $7.66(1.18)$ & 7 & $7.56(0.72)$ & 7 \\
\hline & 9 & Reddish yellow urine $\mathrm{e}^{\mathrm{a}}$ & $7.63(1.01)$ & 7 & $7.59(0.56)$ & 7 \\
\hline & 10 & Sore throat ${ }^{a}$ & $7.56(1.11)$ & 5 & $7.69(0.69)$ & 5 \\
\hline & 11 & Reddish eye $e^{a}$ & $7.56(1.01)$ & 7 & $7.56(0.80)$ & 7 \\
\hline & 12 & Desire to drink ${ }^{\mathrm{a}}$ & $7.56(1.11)$ & 7 & $7.53(0.57)$ & 7 \\
\hline & 13 & Reddened complexion ${ }^{\mathrm{a}}$ & $7.56(0.84)$ & 7 & $7.50(0.72)$ & 6 \\
\hline & 14 & Erythema, blister or ulcer ${ }^{\mathrm{a}}$ & $7.53(0.72)$ & 7 & $7.47(0.98)$ & 7 \\
\hline & 15 & Hard bound or dry stool ${ }^{a}$ & $7.50(1.19)$ & 7 & $7.44(0.67)$ & 7 \\
\hline & 16 & Agitation $^{\mathrm{a}}$ & $7.50(1.11)$ & 6 & $7.38(0.71)$ & 6 \\
\hline & 17 & Red lips ${ }^{\mathrm{a}}$ & $7.47(1.02)$ & 6 & $7.63(0.71)$ & 6 \\
\hline & 18 & Sore swollen gum ${ }^{\mathrm{a}}$ & $7.47(1.02)$ & 7 & $7.44(0.76)$ & 6 \\
\hline & 19 & Dry and yellow fur ${ }^{\mathrm{a}}$ & $7.44(0.56)$ & 7 & $7.47(0.62)$ & 8 \\
\hline & 20 & Hotness in chest ${ }^{a}$ & $7.44(1.01)$ & 7 & $7.38(0.55)$ & 7 \\
\hline & 21 & Red tip of tongue ${ }^{a}$ & $7.44(1.05)$ & 7 & $7.38(0.71)$ & 7 \\
\hline & 22 & Red tip and margin of tongue $\mathrm{e}^{\mathrm{a}}$ & $7.44(1.05)$ & 7 & $7.38(0.71)$ & 7 \\
\hline & 23 & Red dot on tongue ${ }^{\mathrm{a}}$ & $7.41(1.07)$ & 8 & $7.41(0.67)$ & 8 \\
\hline & 24 & Floating and rapid pulse $\mathrm{e}^{\mathrm{a}}$ & $7.38(1.18)$ & 7 & $7.41(0.67)$ & 7 \\
\hline & 25 & Dry tongue with little fluid ${ }^{\mathrm{a}}$ & $7.38(1.01)$ & 7 & $7.31(0.64)$ & 7 \\
\hline & 26 & Dry, yellow and white fur ${ }^{\mathrm{a}}$ & $7.38(0.61)$ & 7 & $7.28(0.63)$ & 7 \\
\hline & 27 & Ulcer on tongue $\mathrm{a}^{\mathrm{a}}$ & $7.34(1.26)$ & 6 & $7.31(0.74)$ & 6 \\
\hline & 28 & Aphtha $^{a}$ & $7.34(1.15)$ & 8 & $7.28(0.68)$ & 8 \\
\hline & 29 & Hot feeling around anus ${ }^{\mathrm{a}}$ & $7.31(1.09)$ & 7 & $7.28(1.02)$ & 7 \\
\hline & 30 & Dry lips ${ }^{\mathrm{a}}$ & $7.31(1.38)$ & 7 & $7.16(0.57)$ & 7 \\
\hline & 31 & Profuse sweating ${ }^{a}$ & $7.31(1.00)$ & 8 & $7.06(0.88)$ & 8 \\
\hline & 32 & Purpura $^{\mathrm{a}}$ & $7.31(1.28)$ & 7 & $7.06(0.76)$ & 7 \\
\hline & 33 & String-like and rapid pulse ${ }^{a}$ & $7.28(1.14)$ & 8 & $7.16(0.63)$ & 7 \\
\hline & 34 & Stinky diarrhea & $7.28(1.16)$ & 7 & $6.97(0.61)$ & 7 \\
\hline & 35 & Slippery and rapid pulse $\mathrm{a}^{\mathrm{a}}$ & $7.22(1.16)$ & 7 & $7.22(0.61)$ & 7 \\
\hline & 36 & Hotness in abdomen ${ }^{\mathrm{a}}$ & $7.22(1.21)$ & 7 & $7.19(0.54)$ & 7 \\
\hline & 37 & Dry throat ${ }^{\mathrm{a}}$ & $7.22(1.29)$ & 7 & $7.16(0.57)$ & 6 \\
\hline & 38 & Decreased urination ${ }^{\mathrm{a}}$ & $7.22(1.04)$ & 6 & $7.09(0.69)$ & 6 \\
\hline & 39 & Dysuria $^{\mathrm{a}}$ & $7.22(0.97)$ & 7 & $6.91(0.93)$ & 7 \\
\hline & 40 & Skin wheal ${ }^{a}$ & $7.16(0.92)$ & 8 & $7.16(0.72)$ & 8 \\
\hline & 41 & Yellow phlegm a & $7.13(1.24)$ & 7 & $7.22(0.55)$ & 7 \\
\hline & 42 & Epistaxis $^{\mathrm{a}}$ & $7.13(1.36)$ & 7 & $7.22(0.66)$ & 7 \\
\hline & 43 & Sudden and watery diarrhea ${ }^{a}$ & $7.13(1.01)$ & 7 & $6.97(1.12)$ & 7 \\
\hline & 44 & Yellow snivel $^{\mathrm{a}}$ & $7.06(1.37)$ & 8 & $7.16(0.63)$ & 7 \\
\hline & 45 & Dyschezia $^{a}$ & $7.06(1.22)$ & 6.5 & $7.09(0.64)$ & 6 \\
\hline & 46 & Anguish in heart ${ }^{\mathrm{a}}$ & $7.03(1.26)$ & 7.5 & $7.19(0.64)$ & 7 \\
\hline & 47 & Dry nasal cavity ${ }^{\mathrm{a}}$ & $7.00(1.30)$ & 7 & $7.09(0.53)$ & 7 \\
\hline & 48 & Bitter taste in mouth ${ }^{\mathrm{a}}$ & $7.00(1.34)$ & 7 & $7.06(0.80)$ & 7 \\
\hline & 49 & Rash $^{\mathrm{a}}$ & $6.97(1.40)$ & 7 & $7.03(0.69)$ & 7 \\
\hline & 50 & Bloody stool with pus ${ }^{\mathrm{a}}$ & $6.97(0.93)$ & 7 & $6.97(1.09)$ & 7 \\
\hline & 51 & Difficulty falling asleep ${ }^{a}$ & $6.94(1.32)$ & 7 & $7.09(0.69)$ & 7 \\
\hline & 52 & Delirious speech ${ }^{\mathrm{a}}$ & $6.84(1.42)$ & 8 & $6.72(0.99)$ & 8 \\
\hline & 53 & Gingival bleeding ${ }^{a}$ & $6.81(1.40)$ & 7 & $6.91(1.03)$ & 7 \\
\hline & 54 & Nasal flaring ${ }^{a}$ & $6.75(1.46)$ & 7 & $6.81(1.06)$ & 7 \\
\hline
\end{tabular}


Table 2 continued

\begin{tabular}{|c|c|c|c|c|c|c|}
\hline \multirow[t]{2}{*}{ Syndrome } & \multirow[t]{2}{*}{ No. } & \multirow[t]{2}{*}{ Item } & \multicolumn{2}{|l|}{ Stage 1} & \multicolumn{2}{|l|}{ Stage 2} \\
\hline & & & Mean (SD) & Median & Mean (SD) & Median \\
\hline & 55 & Difficulty maintaining sleep ${ }^{a}$ & $6.72(1.30)$ & 7 & $6.91(0.59)$ & 7 \\
\hline & 56 & Hemoptysis $^{a}$ & $6.69(1.40)$ & 7 & $6.84(0.85)$ & 7 \\
\hline & 57 & Hematuria $^{a}$ & $6.69(1.49)$ & 7 & $6.66(1.10)$ & 7 \\
\hline & 58 & Headache $^{a}$ & $6.56(1.32)$ & 8 & $6.41(0.87)$ & 7 \\
\hline & 59 & Hematemesis ${ }^{a}$ & $6.44(1.26)$ & 8 & $6.44(0.94)$ & 8 \\
\hline & 60 & Breathless $^{a}$ & $6.34(1.26)$ & 7.5 & $6.13(0.94)$ & 7 \\
\hline & 61 & Lethargy ${ }^{a}$ & $6.31(1.60)$ & 7 & $6.31(1.12)$ & 7 \\
\hline & 62 & Tinnitus $^{a}$ & $5.84(1.65)$ & 7 & $5.94(0.91)$ & 7 \\
\hline
\end{tabular}

The translations were mainly according to "WHO International Standard Terminologies on Traditional Medicine in the Western Pacific Region [70]"

SD standard deviation

a The 178 diagnostic items graded as 7.0 and above

detecting the $Q i$ and $X u e$ (blood) status. Five syndromes, except for Zao (dryness) syndrome, comprised pulse examinations for instant diagnostic judgment in $\mathrm{CM}$ practice [68]. Clinical skills in pulse and tongue examinations were important for accurate assessment of signs and symptoms in the SEED scale [69], just as in CM clinical practice.

The 102 diagnostic criteria were checked by inquiry, inspection, olfaction, audition, percussion, palpation, and pulse examination to ensure complete consideration in medical care. Arrangement of these diagnostic items in a more systematic manner along with a designed record format for tongue and pulse examinations would be necessary for clinical practice. Practice manuals proposing the definition, description, and standardized process for each diagnostic criterion have also been developed for correct implementation.

The limitation of homogeneous education backgrounds is inevitable, since we excluded CM clinicians who had only practicing experience, but no postgraduate degrees from participating in the Delphi panel, as some research background would be required for understanding of the Delphi method and the statistical results circulated during the processes. The panelists' average practicing experience of 11.7 years was considered to be a good representation of their clinical experience.

Because the Liu Yin (six excesses) syndromes were general categories, exploratory and confirmatory factor analyses were conducted to derive and validate the underlying structures of the SEED scale and to reveal the correlations among signs and symptoms. Future studies, including clinical observations to avoid item redundancy and to confirm the clinical practice of the SEED scale in infectious diseases, are required.

It's the first scale based on the six excesses ( Liu Yin) and constructed by the Delphi method; the CM experts epitomized the contents of CM literature and journal articles via the process. Future applications of the SEED scale in clinical practice, research and CM education are expected.

\section{Conclusion}

A CM-based SEED scale was developed for the evaluation and diagnosis of infectious diseases based on only signs and symptoms.

\section{Authors' contributions}

YCS conceived and designed the study. PJC, TCL, LLC and JDL analyzed the data. PJC wrote the manuscript. TCL and $\mathrm{CHC}$ revised the manuscript. All authors read and approved the final manuscript.

\section{Author details}

${ }^{1}$ Graduate Institute of Chinese Medicine, School of Chinese Medicine, China Medical University, Taichung, Taiwan. ${ }^{2}$ Department of Traditional Chinese Medicine, Taichung Veterans General Hospital, Taichung, Taiwan. ${ }^{3}$ Graduate Institute of Biostatistics, China Medical University, Taichung, Taiwan. ${ }^{4}$ Feinberg School of Medicine, Northwestern University, Chicago, USA. ${ }^{5}$ School of Nursing, China Medical University, Taichung, Taiwan. ${ }^{6}$ Department of Nursing, China Medical University Hospital, Taichung, Taiwan. ${ }^{7}$ Department of Healthcare Administration, College of Medical and Health Science, Asia University, Taichung, Taiwan.

\section{Acknowledgements}

This project was supported by Grant (number CCMP97-RD-113) from the Committee on Traditional Chinese Medicine and Pharmacy, Department of Health, Executive Yuan, Taiwan. The support of statistic analysis from the Biostatistics Center of China Medical University was also appreciated.

Compliance with ethical guidelines

Competing interests

The authors declare that they have no competing interests.

Received: 9 March 2014 Accepted: 14 September 2015

Published online: 27 October 2015

\section{References}

1. Writing Committee of the WHO Consultation on Clinical Aspects of Pandemic Influenza. Clinical aspects of pandemic 2009 influenza A (H1N1) virus infection. N Engl J Med. 2010;362(18):1708-19. 
2. Miller E, Hoschler K, Hardelid P, Stanford E, Andrews N, Zambon M. Incidence of 2009 pandemic influenza A H1N1 infection in England: a cross-sectional serological study. Lancet. 2010;375(9720):1100-8.

3. Baden LR, Drazen JM, Kritek PA, Curfman GD, Morrissey S, Campion EW. H1N1 Influenza A disease-information for health professionals. N Engl J Med. 2009;360(25):2666-7.

4. Echevarría-Zuno S, Mejía-Aranguré JM, Mar-Obeso AJ, Grajales-Muñiz C, Robles-Pérez E, González-León M, et al. Infection and death from influenza A H1N1 virus in Mexico: a retrospective analysis. The Lancet. 2009;374(9707):2072-9.

5. Gambotto A, Barratt-Boyes SM, de Jong MD, Neumann G, Kawaoka Y. Human infection with highly pathogenic H5N1 influenza virus. Lancet. 2008;371(9622):1464-75.

6. Webster RG, Govorkova EA. H5N1 influenza-continuing evolution and spread. N Engl J Med. 2006;355(21):2174-7

7. Blyth CC, Iredell JR, Dwyer DE. Rapid-test sensitivity for novel swine-origin influenza A (H1N1) virus in humans. N Engl J Med. 2009;361(25):2493.

8. Oner AF, Bay A, Arslan S, Akdeniz H, Sahin HA, Cesur Y, et al. Avian influenza A (H5N1) infection in eastern Turkey in 2006. N Engl J Med. 2006;355(21):2179-85.

9. Beigel JH, Farrar J, Han AM, Hayden FG, Hyer R, de Jong MD, et al. Avian influenza A (H5N1) infection in humans. N Engl J Med. 2005;353(13):1374-85.

10. Abdel-Ghafar AN, Chotpitayasunondh T, Gao Z, Hayden FG, Nguyen DH, de Jong MD, et al. Update on avian influenza A (H5N1) virus infection in humans. N Engl J Med. 2008;358(3):261-73.

11. Glezen WP. Prevention and treatment of seasonal influenza. N Engl J Med. 2008;359(24):2579-85

12. Stamm W, Wagner K, Amsel R, Alexander E, Turck M, Counts G, et al. Causes of the acute urethral syndrome in women. N Engl J Med. 1980;303(8):409-15

13. Richards D, Toop L, Chambers S, Fletcher L. Response to antibiotics of women with symptoms of urinary tract infection but negative dipstick urine test results: double blind randomised controlled trial. BMJ. 2005;331(7509):143-6

14. Poehling KA, Edwards KM, Weinberg GA, Szilagyi P, Staat MA, Iwane MK, et al. The underrecognized burden of influenza in young children. N Engl J Med. 2006;355(1):31-40.

15. Lessler J, Reich NG, Brookmeyer R, Perl TM, Nelson KE, Cummings DA. Incubation periods of acute respiratory viral infections: a systematic review. Lancet Infect Dis. 2009;9(5):291-300.

16. Tang JL, Liu BY, Ma KW. Traditional Chinese medicine. Lancet. 2008;372(9654):1938-40.

17. Peng J. Historical studies of the systems of diagnosis and treatment of exopathogenic diseases. Zhonghua Yi Shi Za Zhi. 1999;29(2):70-3.

18. Scheid V. The globalization of Chinese medicine. Lancet. 1999;354 (Suppl:SIV10).

19. Zhang GG, Bausell B, Lao L, Handwerger B, Berman BM. Assessing the consistency of traditional Chinese medical diagnosis: an integrative approach. Altern Ther Health Med. 2003;9(1):66-71.

20. Zhang GG, Lee W, Bausell B, Lao L, Handwerger B, Berman B. Variability in the traditional Chinese medicine (TCM) diagnoses and herbal prescriptions provided by three TCM practitioners for 40 patients with rheumatoid arthritis. J Altern Complement Med. 2005;11(3):415-21.

21. Kalauokalani D, Sherman KJ, Cherkin DC. Acupuncture for chronic low back pain: diagnosis and treatment patterns among acupuncturists evaluating the same patient. South Med J. 2001;94(5):486-92.

22. Birch S, Sherman K. Zhong Y acupuncture and low-back pain: traditional Chinese medical acupuncture differential diagnoses and treatments for chronic lumbar pain. J Altern Complement Med. 1999;5(5):415-25 (discussion 27-8)

23. Lindahl MG, Barrett R, Peterson D, Zheng L, Nedrow A. Development of an integrative patient history intake tool: a Delphi study. Altern Ther Health Med. 2005;11(1):52-6.

24. Liu GP, Wang YQ, Dong Y, Zhao NQ, Xu ZX, Li FF, et al. Development and evaluation of an inquiry scale for diagnosis of heart system syndromes in traditional Chinese medicine. Zhong Xi Yi Jie He Xue Bao. 2009;7(1):20-4.

25. Schnyer RN, Conboy LA, Jacobson E, McKnight P, Goddard T, Moscatelli F et al. Development of a Chinese medicine assessment measure: an interdisciplinary approach using the Delphi method. J Altern Complement. 2005:11(6):1005-13.
26. Hogeboom CJ, Sherman KJ, Cherkin DC. Variation in diagnosis and treatment of chronic low back pain by traditional Chinese medicine acupuncturists. Complement Ther Med. 2001;9(3):154-66.

27. Sherman KJ, Cherkin DC, Hogeboom CJ. The diagnosis and treatment of patients with chronic low-back pain by traditional Chinese medical acupuncturists. J Altern Complement Med. 2001;7(6):641-50.

28. Coeytaux RR, Chen W, Lindemuth CE, Tan Y, Reilly AC. Variability in the diagnosis and point selection for persons with frequent headache by traditional Chinese medicine acupuncturists. J Altern Complement Med. 2006;12(9):863-72.

29. Fitch K, Bernstein SJ, Aguilar MD, Burnand B, Lacalle JR, Lazaro P. The RAND/UCLA appropriateness method user's manual: Natl Book Network 2000.

30. Dixon AE, Sugar EA, Zinreich SJ, Slavin RG, Corren J, Naclerio RM, et al. Criteria to screen for chronic sinonasal disease. Chest. 2009;136(5):1324-32.

31. Zhang W, Doherty M, Leeb BF, Alekseeva L, Arden NK, Bijlsma JW, et al. EULAR evidence-based recommendations for the diagnosis of hand osteoarthritis: report of a task force of ESCISIT. Ann Rheum Dis. 2009;68(1):8-17.

32. Sherman PM, Hassall E, Fagundes-Neto U, Gold BD, Kato S, Koletzko S, et al. A global, evidence-based consensus on the definition of gastroesophageal reflux disease in the pediatric population. Am J Gastroenterol. 2009;104(5):1278-95 (quiz 96).

33. Ferri $C P$, Prince $M$, Brayne C, Brodaty $H$, Fratiglioni L, Ganguli M, et al. Global prevalence of dementia: a Delphi consensus study. The Lancet. 2005;366(9503):2112-7.

34. Gallagher P, Ryan C, Byrne S, Kennedy J, O'Mahony D. STOPP (screening tool of older person's prescriptions) and START (screening tool to alert doctors to right treatment) consensus validation. Int J Clin Pharmacol Ther. 2008;46(2):72-83.

35. Bowles KH, Holmes JH, Ratcliffe SJ, Liberatore M, Nydick R, Naylor MD. Factors identified by experts to support decision making for post acute referral. Nurs Res. 2009;58(2):115-22.

36. McGlynn EA, Asch SM, Adams J, Keesey J, Hicks J, DeCristofaro A, et al. The quality of health care delivered to adults in the United States. N Engl J Med. 2003;348(26):2635-45

37. Koekkoek B, van Meijel B, Schene A, Hutschemaekers G. A Delphi study of problems in providing community care to patients with nonpsychotic chronic mental illness. Psychiatr Serv. 2009;60(5):693-7.

38. Jha AK, DesRoches CM, Campbell EG, Donelan K, Rao SR, Ferris TG, et al. Use of electronic health records in US hospitals. N Engl J Med. 2009;360(16):1628-38

39. Tierney S, Fox JR. Chronic anorexia nervosa: a Delphi study to explore practitioners' views. Int J Eat Disord. 2009;42(1):62-7.

40. Alahlafi A, Burge S. What should undergraduate medical students know about psoriasis? Involving patients in curriculum development: modified Delphi technique. BMJ. 2005;330(7492):633-6.

41. Kassab S, Cummings M, Berkovitz S, van Haselen R, Fisher P. Homeopathic medicines for adverse effects of cancer treatments. Cochrane Database Syst Rev. 2009;(2):CD004845. doi:10.1002/14651858.CD004845.pub2

42. Salliot C, Dernis E, Lavie F, Cantagrel A, Gaudin P, Wendling D, Claudepierre P, Flipo RM, Goupille PM, Le Loët X, Maillefert JF, Paul C, Saraux A, Schaeverbeke T, Tebib J, Combe B. Diagnosis of peripheral psoriatic arthritis: recommendations for clinical practice based on data from the literature and experts opinion. Joint Bone Spine. 2009;76(5):532-9.

43. Taylor WJ, Shewchuk R, Saag KG, Schumacher HR Jr, Singh JA, Grainger R, et al. Toward a valid definition of gout flare: results of consensus exercises using Delphi methodology and cognitive mapping. Arthritis Rheum. 2009;61(4):535-43.

44. Murray PT, Devarajan P, Levey AS, Eckardt KU, Bonventre JV, Lombardi R, et al. A framework and key research questions in AKI diagnosis and staging in different environments. Clin J Am Soc Nephrol. 2008;3(3):864-8.

45. Guellac N, Niehues T. Interdisciplinary and evidence-based treatment guideline for juvenile idiopathic arthritis. Klin Padiatr. 2008;220(6):392-402.

46. Graham B, Regehr G, Wright JG. Delphi as a method to establish consensus for diagnostic criteria. J Clin Epidemiol. 2003;56(12):1150-6.

47. Pastor AC, Osman F, Teitelbaum DH, Caty MG, Langer JC. Development of a standardized definition for Hirschsprung's-associated enterocolitis: a Delphi analysis. J Pediatr Surg. 2009;44(1):251-6. 
48. Cheon JH, Kim ES, Shin SJ, Kim TI, Lee KM, Kim SW, et al. Development and validation of novel diagnostic criteria for intestinal Behcet's disease in Korean patients with ileocolonic ulcers. Am J Gastroenterol. 2009;104(10):2492-9. doi:10.1038/ajg.2009.331.

49. Campos F, Sousa A, Rodrigues V, Marques A, Queiros C, Dores A. Practical guidelines for peer support programmes for mental health problems. Rev Psiquiatr Salud Ment. 2014. doi:10.1016/j.rpsm.2014.06.002.

50. Ferreira JJ, Santos AT, Domingos J, Matthews H, Isaacs T, Duffen J, et al. Clinical parameters and tools for home-based assessment of Parkinson's disease: results from a Delphi study. J Parkinsons Dis. 2015;5(2):281-90.

51. Loeffen EA, Mulder RL, Kremer LC, Michiels EM, Abbink FC, Ball LM, et al. Development of clinical practice guidelines for supportive care in childhood cancer-prioritization of topics using a Delphi approach. Support Care Cancer. 2014. doi:10.1007/s00520-014-2559-7.

52. Munck A, Mayell SJ, Winters V, Shawcross A, Derichs N, Parad R, et al. Cystic fibrosis screen positive, inconclusive diagnosis (CFSPID): a new designation and management recommendations for infants with an inconclusive diagnosis following newborn screening. J Cyst Fibros. 2015 doi:10.1016/j.jcf.2015.01.001.

53. Pucher PH, Brunt LM, Fanelli RD, Asbun HJ, Aggarwal R. SAGES expert Delphi consensus: critical factors for safe surgical practice in laparoscopic cholecystectomy. Surg Endosc. 2015. doi:10.1007/s00464-015-4079-z.

54. Roberts DM, Yates C, Megarbane B, Winchester JF, Maclaren R, Gosselin $\mathrm{S}$, et al. Recommendations for the role of extracorporeal treatments in the management of acute methanol poisoning: a systematic review and consensus statement. Crit Care Med. 2015;43(2):461-72. doi:10.1097/ ccm.0000000000000708.

55. Slade SC, Dionne CE, Underwood M, Buchbinder R. Standardised method for reporting exercise programmes: protocol for a modified Delphi study. BMJ Open, vol. 12. England: BMJ Publishing Group Limited; 2014. p. e006682.

56. Suzuki Y, Fukasawa M, Nakajima S, Narisawa T, Keiko A, Kim Y. Developing a consensus-based definition of "Kokoro-no Care" or mental health services and psychosocial support: drawing from experiences of mental health professionals who responded to the Great East Japan Earthquake. PLoS Curr. 2015. doi:10.1371/currents.dis.cfcbaf509711641ab5951535851 e572e.

57. Trevelyan EG, Turner WA, Robinson N. Developing an acupuncture protocol for treating phantom limb pain: a Delphi consensus study. Acupunct Med, vol. 1. England: BMJ Publishing Group Limited; 2015. p. 42-50.

58. James DJ, Warren-Forward HM. Development of consensus statements for pregnancy screening in diagnostic nuclear medicine: a delphi study. J Nucl Med Technol, vol. 1. United States: The Society of Nuclear Medicine and Molecular Imaging, Inc; 2015. p. 74-9.
59. Kunneman M, Pieterse AH, Stiggelbout AM, Marijnen CA. Which benefits and harms of preoperative radiotherapy should be addressed? A Delphi consensus study among rectal cancer patients and radiation oncologists. Radiother Oncol. 2015;114(2):212-7. doi:10.1016/j.radonc.2014.11.034.

60. Muller BG, van den Bos W, Brausi M, Futterer JJ, Ghai S, Pinto PA, et al. Follow-up modalities in focal therapy for prostate cancer: results from a Delphi consensus project. World J Urol. 2015. doi:10.1007/ s00345-014-1475-2.

61. Paneque $M$, Sequeiros J, Skirton $H$. Quality issues concerning genetic counselling for presymptomatic testing: a European Delphi study. Eur J Hum Genet. 2015. doi:10.1038/ejhg.2015.23

62. Strosberg JR, Fisher GA, Benson AB, Anthony LB, Arslan B, Gibbs JF, et al. Appropriateness of systemic treatments in unresectable metastatic welldifferentiated pancreatic neuroendocrine tumors. World J Gastroenterol. 2015;21(8):2450-9. doi:10.3748/wjg.v21.i8.2450.

63. Temkin-Greener H, Ladwig S, Caprio T, Norton S, Quill T, Olsan T, et al. Developing palliative care practice guidelines and standards for nursing home-based palliative care teams: a Delphi study. J Am Med Dir Assoc 2015;16(1):86 e1-7. doi:10.1016/j.jamda.2014.10.013.

64. Vilches-Moraga A, Arino-Blasco S, Verdejo-Bravo C, Mateos-Nozal J. University studies plan in geriatric medicine developed using a modified Delphi technique. Rev Esp Geriatr Gerontol. 2014. doi:10.1016/j. regg.2014.10.003.

65. Fitch K, Bernstein SJ, Aguilar MD, Burnand B, LaCalle JR, Lazaro P, et al. The RAND/UCLA appropriateness users manual. Santa Monica: RAND Institute; 2001.

66. Anonymous. Huangdi's internal classic, before Han dynasty.

67. Hsiao CF, Tsou HH, Wu YJ, Lin CH, Chang YJ. Translation in different diagnostic procedures - traditional Chinese medicine and Western medicine. J Formos Med Assoc. 2008;107(12 Suppl):74-85.

68. O'Brien KA, Abbas E, Zhang J, Guo ZX, Luo R, Bensoussan A, et al. Understanding the reliability of diagnostic variables in a Chinese Medicine examination. J Altern Complement Med. 2009;15(7):727-34.

69. Chiang PJ, Su YC, Liou DM. Acupuncture for chronic low back pain. N Engl J Med. 2010;363(18):1775-6 author reply 77-8.

70. WHO Regional Office for the Western Pacific. WHO international standard terminologies on traditional medicine in the Western Pacific Region. 2007.

\section{Submit your next manuscript to BioMed Central and take full advantage of:}

- Convenient online submission

- Thorough peer review

- No space constraints or color figure charges

- Immediate publication on acceptance

- Inclusion in PubMed, CAS, Scopus and Google Scholar

- Research which is freely available for redistribution

Submit your manuscript at 